\title{
A Dry Electrode Cap and Its Application in a Steady-State Visual Evoked Potential-Based Brain-Computer Interface
}

\author{
Xiaoting $\mathrm{Wu}^{1,2}$, Li Zheng ${ }^{1,2}$, Lu Jiang ${ }^{1,2}$, Xiaoshan Huang ${ }^{3}$, Yuanyuan Liu ${ }^{2,4}$, Lihua Xing 5,6, \\ Xiao Xing ${ }^{1,2}$, Yijun Wang 1,2,7, Weihua Pei ${ }^{1,2,7, *}$, Xiaowei Yang ${ }^{1}$, Zhiduo Liu ${ }^{1,2}$, \\ Chunrong Wei ${ }^{1,2}$, Yamin Li ${ }^{1,2}$, Miao Yuan ${ }^{1,2}$ and Hongda Chen ${ }^{1,2}$ \\ 1 The State Key Laboratory on Integrated Optoelectronics, Institute of Semiconductors, \\ Chinese Academy of Sciences, Beijing 100083, China; wuxiaoting@semi.ac.cn (X.W.); \\ zhengli17@semi.ac.cn (L.Z.); jianglu@semi.ac.cn (L.J.); xingxiao@cert.org.cn (X.X.); wangyj@semi.ac.cn (Y.W.); \\ yangxiaowei@semi.ac.cn (X.Y.); liuzhiduo@semi.ac.cn (Z.L.); weichunrong@semi.ac.cn (C.W.); \\ liyamin_1994@126.com (Y.L.); yuanmiao@semi.ac.cn (M.Y.); hdchen@semi.ac.cn (H.C.) \\ 2 University of Chinese Academy of Sciences, Beijing 100049, China; liuyy@semi.ac.cn \\ 3 Neuracle Technology company, Beijing 100097, China; hxs@neuracle.cn \\ 4 Engineering Research Center for Semiconductor Integrated Technology, Institute of Semiconductors, \\ Chinese Academy of Sciences, Beijing 100083, China \\ 5 Beijing minghao intelligent design company, Beijing 100085, China; 13641011339@139.com \\ 6 Beijing bioblue sensor company, Beijing 100085, China \\ 7 CAS Center for Excellence in Brain Science and Intelligence Technology, Shanghai 200031, China \\ * Correspondence: peiwh@semi.ac.cn; Tel.: +86-010-82304331
}

Received: 30 August 2019; Accepted: 20 September 2019; Published: 23 September 2019

\begin{abstract}
The wearable electroencephalogram (EEG) dry electrode acquisition system has shown great application prospects in mental state monitoring, the brain-computer interface (BCI), and other fields due to advantages such as being small in volume, light weight, and a ready-to-use facility. This study demonstrates a novel EEG cap with concise structure, easy adjustment size, as well as independently adjustable electrodes. The cap can be rapidly worn and adjusted in both horizontal and vertical dimensions. The dry electrodes on it can be adjusted independently to fit the scalp as quickly as possible. The accuracy of the BCI test employing this device is higher than when employing a headband. The proposed EEG cap makes adjustment easier and the contact impedance of the dry electrodes more uniform.
\end{abstract}

Keywords: adjustable dry electrodes EEG cap; brain-computer interface; steady-state visual evoked potential

\section{Introduction}

The brain-computer interface (BCI), especially the electroencephalogram (EEG)-based non-invasive $\mathrm{BCI}$, is a promising human-computer interaction technology [1]. The EEG signal acquisition system plays a significant role in BCI technology. Generally speaking, the EEG acquisition system is composed of electrodes, a cap or headset, amplification circuits, and a wire or wireless transmission module. A ready-to-use EEG cap is a decisive technology if BCI will be extended from the laboratory to practical applications. Dry electrodes need no gel nor cleaning after use. It might be an ideal EEG sensor device if it had electrical characteristic as good as a wet electrode [2-4]. However, the electrical connection between the dry electrode and scalp is not good due to the absence of the conductive paste buffer layer. Many methods are used to improve the convenience and the electrical characteristics of dry electrode systems. At present, few dry EEG caps can behave like their wet opponent $[5,6]$. There are still many problems to be solved. 
A stable electrical connection between the scalp and the dry electrode is the base of a high-quality EEG recording. A proper press can tightly mount the dry electrode on the head with less uncomfortability. It is a great challenge to meet these requirements on the hair-covered scalp. Therefore, an EEG cap often adopts an elastic supporting-structure on the back of the dry electrode to provide proper press. For example, g. SAHARA dry electrodes are mounted on an elastic cloth cap named the g. GAMMA cap [7]. A wireless EEG acquisition device produced by the Emotive company $[1,8]$ utilizes elastic shafts to compose a hand-shaped electrode-mounting structure and the electrodes are fixed at the end of the fingers. A prototype of a three-channel dry electrodes EEG system developed by Brain Products was introduced to a BCI application [2]. A Cognionics 64-channel mobile EEG system was used for online neuroimaging and state classification [9]. Other similar EEG acquisition devices, such as elastic headbands, have also been developed in $[3,10,11]$ or produced by Advanced Brain Monitoring. Besides these elastic structures on the cap, spring or elastic structures are used on the dry electrode as reported in [12-14]. These kinds of elastic supporting structures or dry electrode structures provide great convenience and have a comfortable feeling. However, elastics could not solve all the problems. Many works explored the usability of dry EEG electrodes in order to achieve a more user-friendly system $[4,15,16]$, but without the aid of gel, the contact impedance and signal-to-noise ratio of the dry electrode largely depended on the contact area between the electrode and the scalp, namely, the pressure applied upon the electrode [17]. Furthermore, head shape and size are different from one person to another. Each electrode makes contact with the head on a different location and from a different angle. All the electrodes on one cap are hard to be placed well at once. Take the elastic cloth cap or the elastic headband as an example, when the cap is worn, more or fewer electrodes need adjusting to reduce the impedance to a certain range. Since all the electrodes are fixed on the same substrate, when one electrode is tight, the other one around it is loose. A common phenomenon is one poor connecting electrode is replaced by other poor connecting electrodes. It is hard to ensure that all the dry electrodes on one cap have acceptable impedance under the proper press. Another shortcoming of these kinds of EEG caps is the electrodes' adjusting is done by an assistant in most cases. Except if the subject himself or herself is familiar with the EEG cap.

To address this deficiency, a prototype of a dry electrode cap was proposed and demonstrated in this study. Compared with cloth caps or headbands, knobs and screws on the proposed cap provide a simple and intuitive adjusting method. The independently adjusting mechanism on each electrode makes adjusting more efficient. The location of eight electrodes referring to the international 10-10 system were placed at the occipital area. The cap was designed to acquire steady-state visual evoked potential (SSVEP) signals. SSVEP is widely used as a BCI method because of its ease of use and needing less training [18-21]. The classification accuracy of the BCI application was employed to evaluate the performance of the proposed device.

\section{Design and Preparation}

\subsection{EEG Cap}

Different from wet electrodes, which can use the conductive paste to fill the gap and decrease the impedance between the scalp and electrode, the dry electrode must contact the scalp directly. If an adjusting device is placed on each electrode on the cap, the gap will be eliminated by adjusting the height of the electrode and, meanwhile, the contact between the electrode and the scalp can be improved.

A skeleton-structure-cap combined with a removable electrode panel was designed to reduce the weight and increase the flexibility, as shown in Figure 1a. The cap mainly consisted of a horizontal hoop and a semi-circular hoop perpendicular to the horizontal one. The hoops were fabricated from polyurethane, a flexible but almost non-elastic polymer material. With the help of a rack-and-pinion structure, the length of the hoops can be adjusted to fit a different head circumference by turning a rotary knob on them, either horizontally or vertically. The electrode panel, as shown in Figure 1b, is a curve plate onto which the adjustable dry electrodes and a wireless EEG acquisition module [22] 
are assembled. The module, as shown in the dotted box in Figure $1 \mathrm{~b}, \mathrm{~d}$, is a commercial instrument including an amplifier, wireless transfer, and EEG recording provided by the Neuracle technology company. The electrode panel's size and shape were customized according to the recording brain area. In this embodiment, the electrode panel was designed and 3D printed with photosensitive resin to put on the occipital region, where the visual cortex is located, to record SSVEP. Eight holes with nuts, on the occipital region (PO1, PO3, POz, PO4, $\mathrm{PO} 2, \mathrm{O} 1, \mathrm{Oz}$, andO2) referring to the international 10-10 syetem, were reserved for the height adjustable dry electrodes. The reference and ground electrodes were placed at the left and right mastoid behind the ears, respectively.

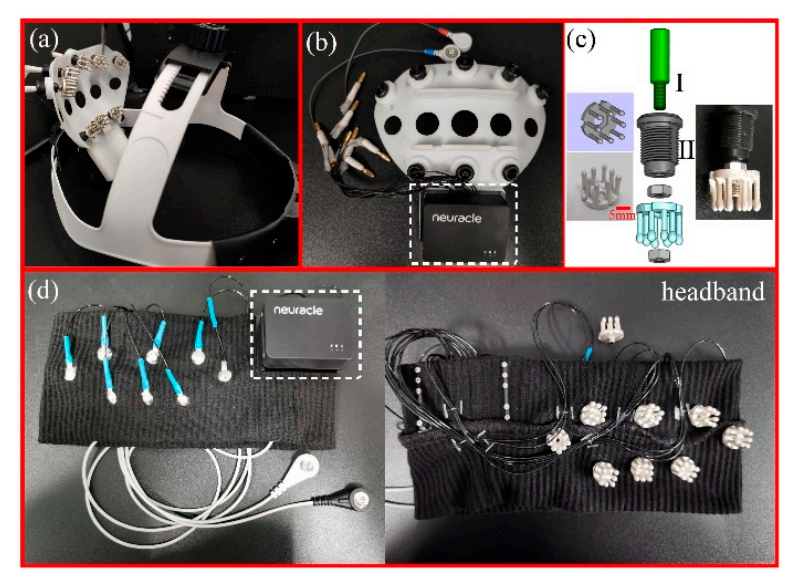

Figure 1. An adjustable electroencephalogram (EEG) cap and headband. (a) Photograph of the EEG cap; (b) the electrode panel with the eight adjustable electrodes and wireless acquisition module (as framed by the dotted line) on it; (c) the multi-pin dry electrode and a mechanically adjustable and electric connection structure, where part I is a tubular socket, part II is a hollow screw, and the left insert is the fabricated electrode and the right insert is the adjustable electrode; and (d) the textile headband for comparison. The dry electrodes and the wireless acquisition module are the same as those used for the cap.

\subsection{Adjustable Dry Electrode}

A kind of multi-pin dry electrode fabricated in our laboratory was employed in this paper. The photograph of the device was shown in the left inset in Figure 1c. The diameter of the dry electrode is $14 \mathrm{~mm}$. There are 8 pins in one electrode. The height and the width of the pin are $8 \mathrm{~mm}$ and $1 \mathrm{~mm}$, respectively. Stereolithography (SLA) 3D printing was used to fabricate the substrate of the electrodes. After the substrate had been completely cleaned and activated by wet chemistry and oxygen plasma, a layer of conductive silver paste was covered by dip coating and cured at $100^{\circ} \mathrm{C}$ for $1 \mathrm{~h}$. The detailed fabrication process can be found in Reference [3].

A mechanically adjustable and electric connection handle was designed and developed for the dry electrode. The handle is mainly composed of two parts. The schematic diagram of the structure was shown in Figure 1c. Part I is a tubular socket made of brass (marked I in Figure 1c). On one end a screw was used to install the dry electrode. The other end is a hollow tube used as a socket. The outside surface of the hollow end was smooth. Part II is a hollow screw made of phenolic resin (marked II in Figure 1c). Its inside surface was smooth too. Only two steps were needed to prepare an adjustable dry electrode. Firstly, insert the screw end of part I into the hole of part II. Part I can freely rotate inside part II along the axis. Secondly, install the dry electrode on part II with two nuts. A handled dry electrode was shown in the right inset in Figure 1c. Then, it can be installed on the cap through the assembly of screw on the part II and nut on the electrode panel, as shown in Figure 1a. The electrode will move downwards or upwards by turning the screws on part II. The thread length of the screw is $10 \mathrm{~mm}$. The adjustable length is nearly $7 \mathrm{~mm}$. When the front end of the dry electrode makes contact with the scalp, the dry electrode, and its electrical connection, will not turn around with the screw. Only up or down movement is retained. This adjustable structure has two merits. One is 
that the dry electrode will not produce tangential friction on the scalp. The other is that the electrical contact states of electrode other than the adjusting one are rarely affected.

A contact pin fit to the socket on part I was used to connect the dry electrodes to the wireless EEG acquisition module circled by a dotted line as shown in Figure $1 \mathrm{~b}$,d. The module is battery powered and the recorded data are transmitted by Wi-Fi technology in real-time. A dedicated router and computer were used to receive, record, and process the data. The contact impedance of every electrode can be detected and displayed on the screen in real-time after the module is turned on.

An elastic textile cap or flexible strip is a widely used device to fix electrodes on the head, as reported in $[5,6,23,24]$. In this paper, an elastic textile headband with the identical dry electrodes, amplifier, and acquisition system was prepared for comparison, as shown in Figure 1d. Eight dry electrodes, with the corresponding location as that of the cap, were riveted on the headband and welded to the amplifier.

\section{Performance Test}

The dry EEG cap and the headband were worn and tested for comparison on twelve volunteers. A test session included performance in BCI task, the impedance of electrodes, as well as evaluation of comfort level. Each volunteer was asked to conduct the test session twice. One was with the cap, the other was with the headband. The interval time between two test sessions was about $30 \mathrm{~min}$. The EEG cap or the headband was worn in random order. The subjects were college students including nine females with long hair and three males with short hair. Their ages ranged from 22 to 29 . All of the subjects were healthy and had a normal or corrected-to-normal vision. Informed consent was obtained from all participants. No scalp cleaning and special preparation were done on the scalp before each test.

One can easily wear the EEG cap even by himself or herself. First, the subject loosens the hoops by turning the rotary knobs on it, set it on the head, and fasten the hoops. At this time, some of the dry electrodes will contact the scalp. Second, the experimenter is responsible for adjusting the electrodes by checking the impedance value and turning the handle of the dry electrode; the impedance of each dry electrode can be adjusted quickly. Figure 2a,b showed the back and side view of the EEG cap worn by a volunteer.

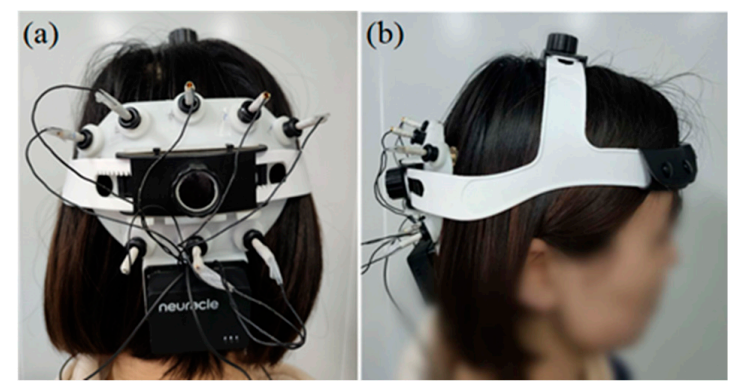

Figure 2. The proposed EEG cap on the head. (a) Back view and (b) side view.

A pre-experiment was arranged to certify the performance of the proposed cap. The proposed cap was compared with a commercial EEG cap, DSI-24 headset [25], which is produced by Wearable Sensing LLC (San Diego, CA, USA) with 21 dry electrodes (Fp1, Fp2, Fz, F3, F4, F7, F8, Cz, C3, C4, T7/T3, T8/T4, Pz, P3, P4, P7/T5, P8/T6, O1, O2, A1, and A2 according to the international 10-20 system) on it. Due to the different distributions of electrodes on the commercial cap and the proposed cap, four electrodes with close locations were chosen from the commercial cap (O1, O2, P3, and P4) and the proposed cap (O1, O2, PO3, and PO4), respectively, to compare the quality of the signal. Ten out of the twelve volunteers attended the pre-experiment. SSVEP was acquired and the signal-to-noise ratio (SNR) was defined as the ratio of the spectrum amplitude at stimulation frequency and the mean value of the two neighboring frequencies. 


\subsection{Performance in $B C I$}

In the experiment, the SSVEP data were acquired and analyzed to find the effects of electrode fixture on the accuracy of the BCI. After being worn, the dry electrodes on the cap or on the headband were adjusted to achieve lower impedance by monitoring the contact impedance in real-time. Since the cap or the headband was designed as a fast and convenient device, comfort of wearing and time spent on wearing must be considered. For practicality and comparison purpose, the adjustment of each wearing was required to be ended in $3 \mathrm{~min}$ by the experimenter. After adjustment, the EEG signals were amplified, analog to digital converted, and wirelessly transferred to a data recording system at a sampling rate of $1000 \mathrm{~Hz}$.

In the experiment, a $3 \times 4$ matrix consisting of 12 flickering stimuli was displayed on a $24.5 \mathrm{inch}$ screen with a refresh rate of $60 \mathrm{~Hz}$. Each stimulus target was a $122 \mathrm{~mm} \times 83 \mathrm{~mm}$ rectangle with a frequency-phase coded method. The frequency ranged from $9 \mathrm{~Hz}$ to $12 \mathrm{~Hz}$ with an interval of $0.25 \mathrm{~Hz}$ except for $10 \mathrm{~Hz}$, and the phase started from 0 to $1.5 \pi$ with an interval of $0.5 \pi$. During the experiment, the subject was seated $0.5 \mathrm{~m}$ away from the screen. Each experiment consisted of 4 blocks and each block contained 48 trials (12 stimulus targets traversed 4 times in random order). Each trial began with a 1 s-long visual cue to point out the target to gaze. Then, all the targets started to flash for $2 \mathrm{~s}$. The subject should move his or her gaze to the target and keep their eyes on it during this time. After the flash, a 1 s-long feedback with a red box will appear, and at the same time, the corresponding text in the red box will appear in the upper left of the screen, as shown in Figure 3. Each trial lasted for $4 \mathrm{~s}$, and 1 block lasted for $3.2 \mathrm{~min}$. To prevent visual fatigue, each subject was required to rest for about 15 min between two blocks.

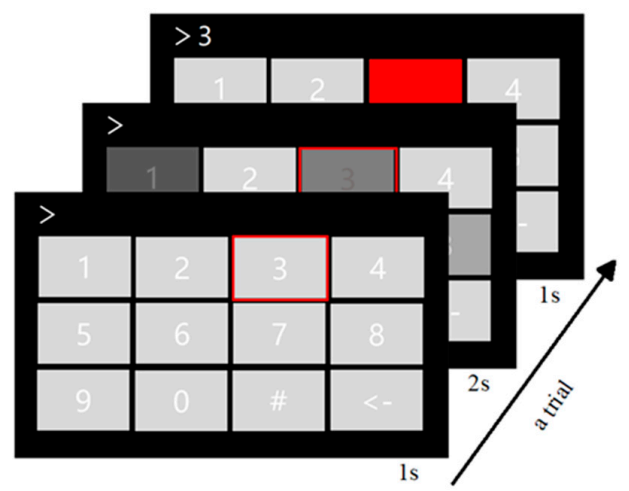

Figure 3. The timeline of a trial in the steady-state visual evoked potential (SSVEP)-based brain-computer interface (BCI) experiment, including visual cues with a red frame for $1 \mathrm{~s}$, stimulus with a red frame for $2 \mathrm{~s}$, and feedback with a red box for $1 \mathrm{~s}$.

The acquired EEG data were first down-sampled to $250 \mathrm{~Hz}$, and the accuracy of the system was analyzed using data epochs of $2 \mathrm{~s}$ after the stimulation onset. After extracting the epoch, the data were preprocessed by a filter bank which was applied to decompose SSVEPs into sub-band components so that the information embedded in the harmonic components can be extracted more efficiently [26]. The task-related component analysis (TRCA) algorithm [27] was used for feature extraction. TRCA can construct spatial filters to extract task-related components, and then Pearson's correlation coefficients between the single-trial and all types of averaged individual templates were calculated. The classification result was determined by the target with the largest correlation coefficient. The system performance was evaluated using classification accuracy. The quality of the EEG is the key to the communication speed of the BCI. Data with a higher signal-to-noise ratio (SNR) require shorter lengths for accurate target identification, leading to increased information transfer rates (ITRs). Shorter data and higher accuracy correspond to a better quality of the SSVEP signal $[3,28]$. 


\subsection{Impedance Test}

The impedance of every electrode was tested near $10 \mathrm{~Hz}$. A $48 \mathrm{nA}$ peak-to-peak square wave current at $7.8 \mathrm{~Hz}$ was used to drive the loop of the ground and the dry electrodes. The impedance value of each channel was calculated by monitoring the voltage difference between the working and the reference electrode. The impedance testing function was integrated into the acquisition module. The work states of the module can be switched by software between "impedance testing" and "EEG recording". The impedance values of each electrode in each experiment was recorded before BCI testing.

\subsection{Comfort Level Assessing}

The comfort level is an important element for the practical application. Therefore, a comfort rate ranking was arranged in the experiment. The subjects were asked to fill in a questionnaire on which four comfort levels were listed. Level 1 represents very comfortable, 2 represents comfortable, 3 represents uncomfortable, and 4 represents uncomfortable with little pain. Subjects were required to fill the questionnaire before and after the BCI test when they were wearing the headband or the EEG cap.

To make sure how the impedance value affects the accuracy of the BCI, five out of twelve volunteers were recruited. These subjects sacrificed a little comfort for low impedance. The criterion of the impedance adjustment on these volunteers was fastening the knob or the screw on the cap until they feel uncomfortable. This situation is called "tight contact", and another comfortable situation is called "loose contact". Then, the impedance and BCI performance of these five subjects were compared in both aforementioned situations.

\section{Results and Discussion}

\subsection{EEG Signal Quality}

Figure 4 shows the narrowband filtered time-domain waveforms and spectrum of a typical steady-state visual evoked EEG signal extracted from the same subjects on the O1 position at a stimulation frequency of $12 \mathrm{~Hz}$ in the pre-experiment. The average SNR of ten subjects with the commercial cap is $10.43 \mathrm{~dB}$ and is $10.13 \mathrm{~dB}$ with the proposed cap. The t-test value of ten subjects' SNR at $12 \mathrm{~Hz}$ between these two caps $(p=0.8835)$ indicated no significant difference and verified that the cap developed in the study had an equal SNR with the commercial cap and can work as well as that of the commericial cap.
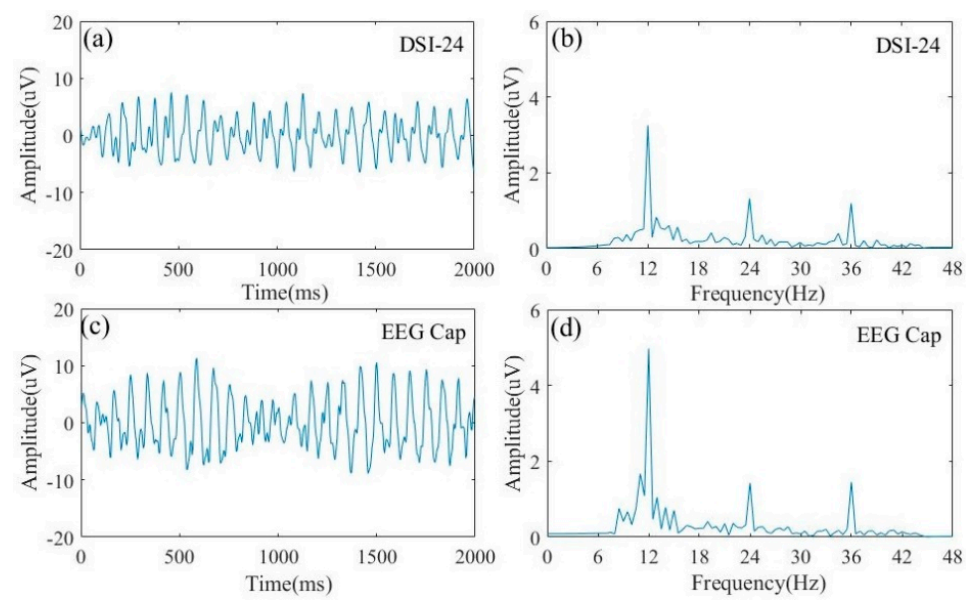

Figure 4. A typical stable visual evoked EEG waveform (a) and its spectrum (b) at $12 \mathrm{~Hz}$ recorded by the DSI-24. A typical stable visual evoked EEG waveform (c) and its spectrum (d) at $12 \mathrm{~Hz}$ recorded by the EEG cap. 


\subsection{BCI Test Results}

The data length (from $0.2 \mathrm{~s}$ to $2 \mathrm{~s}$ ) used in the TRCA method versus the accuracy was shown in Figure 5. The accuracy rate of either the cap or the headband raised as the data length increased. The accuracy of the cap was always higher than that of the headband at a different data length. The mean classification accuracy with a $2 \mathrm{~s}$ data length can reach to 0.9753 with the proposed EEG cap, higher than 0.9575 with the headband for 12 subjects. The statistical significance test of the accuracy between the EEG cap and the headband indicated that the cap and the headband have a significance difference $(p=0.00028)$, namely, the EEG quality, or signal-to-noise ratio of EEG of the proposed cap was better than that of the contrast headband. The performance of five out of twelve students in both "tight contact" and "loose contact" situations was calculated. The classification accuracy of these two states are 0.9885 and 0.9875 with a $2 \mathrm{~s}$ data length, and the t-test results $(p=0.0503)$ indicated no significant difference between these two states. The average accuracy of five subjects under the loose contact situation is higher than the average accuracy of twelve subjects under the loose contact situation, which is caused by the the fluctuation of fewer samples. The results tend to be consistent if the volume of sampling is enough.

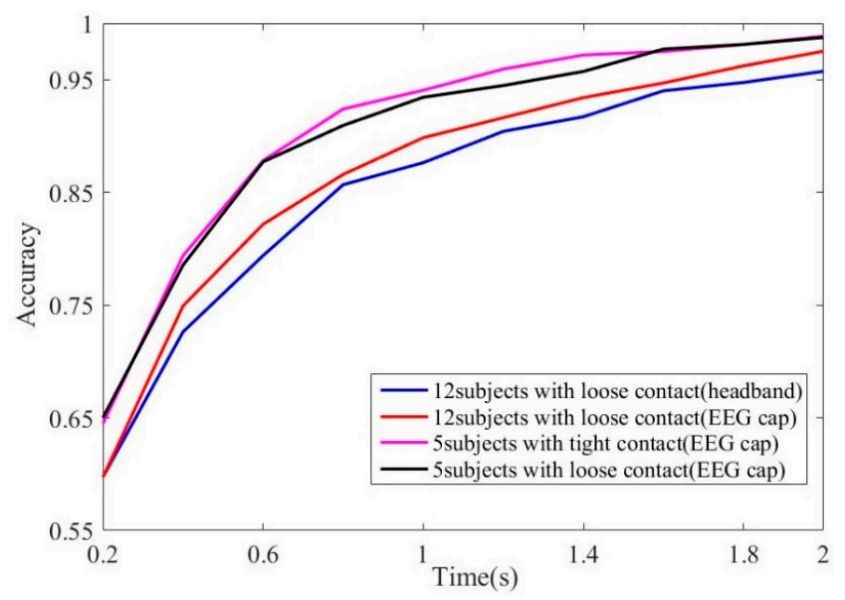

Figure 5. The results of the classification accuracy rate of the headband and the proposed EEG cap with different data lengths. The pink line represents the mean accuracy of five subjects under tight contact with the EEG cap; the black line represents the mean accuracy of five subjects under loose contact with the EEG cap; the red line represents the mean accuracy of twelve subjects under loose contact with the EEG cap; and the blue line represents the mean accuracy of twelve subjects under loose contact with the headband.

\subsection{Impedance and Comfort-Level Assessing}

The impedance value distribution of the cap and the headband of twelve subjects were shown in Figure $6 a, b$, respectively. The impedance of $51 \%$ electrodes on the headband was larger than $1000 \mathrm{~K} \Omega$. The ratio of electrodes with an impedance lower than $100 \mathrm{~K} \Omega$ was $2 \%$. The corresponding percentages on the cap were $31 \%$ and $6 \%$. Compared with the headband, the cap had a lower average impedance. As far as the impedance distribution was concerned, the cap can get a more uniform impedance value. From the results above, we noticed that the average impedance of the EEG cap was high, although the cap acquired effective and reliable EEG data for a SSVEP-based BCI. We inferred that loose and comfortable wearing of the cap and headband resulted in high impedance. Therefore, we recorded the impedance distributions of the five subjects in "tight contact" and "loose contact" situations as shown in Figure $6 c$,d. It can be clearly seen that high pressure can decrease the impedance. The ratio of electrodes with an impedance lower than $100 \mathrm{~K} \Omega$ was $22 \%$ and no electrode's impedance was higher than $1000 \mathrm{~K} \Omega$ in the "tight contact" situation. The lowest impedance was only $13 \mathrm{~K} \Omega$. However, the corresponding ratios were $7 \%$ and $35 \%$ in the "loose contact" situation. All in all, 
higher pressures resulted in lower and more uniform impedance, but the BCI performance was not significantly improved.

Impedance distribution

(a)

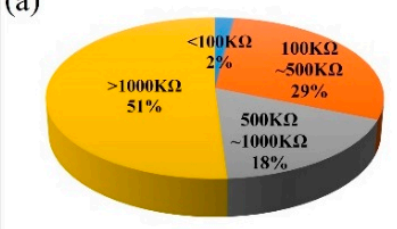

12 subjects with loose contact (headband)

(c)

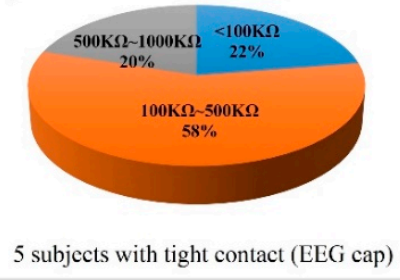

(b)

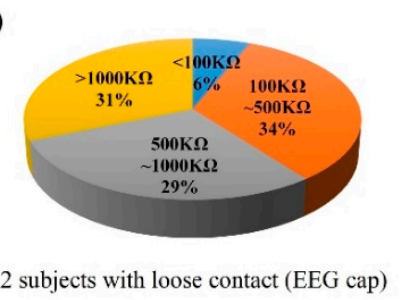

(d)

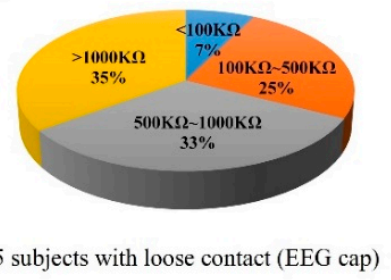

Figure 6. The impedance value distribution of (a) 12 subjects under loose contact with a headband; (b) 12 subjects under loose contact with the EEG cap, (c) 5 subjects under tight contact with the EEG cap (d); and 5 subjects under loose contact with the EEG cap.

According to these results, we calculated the Pearson's coefficient between the impedance and accuracy of the twelve subjects. The results showed that neither the headband $(\mathrm{R}=0.0838, p=0.7958)$ nor the EEG cap $(\mathrm{R}=-0.4056, p=0.1909)$ exhibited a significant correlation between accuracy and impedance. Given to the usability of the EEG cap, a balance between performance and comfort must be obtained.

The comfort level assessesment results are shown in Figure 7. It can be seen that both the cap and the headband showed no significant uncomfortable feelings before BCI testing, while after the experiment, the proposed EEG cap may cause an uncomfortable feeling due to its inelastic polymer material.

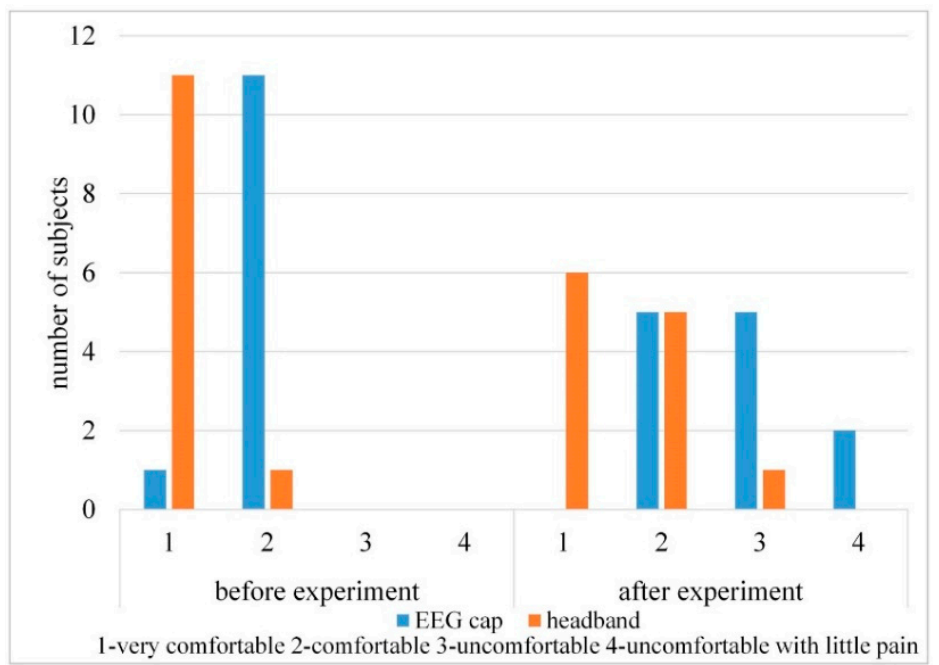

Figure 7. Comfort assessment of the EEG cap and headband before and after the experiment. Level 1 represents very comfortable, 2 represents comfortable, 3 represents uncomfortable, and 4 represents uncomfortable with little pain. 
Compared with the headband, the adjustable cap provided more options to the subjects, wearing the electrode loosely or tightly. To the subjects unfamiliar with EEG acquisition, the knob and screws on the cap make the adjustment easier. A headband can only provide a constant elastic degree. Adjusting the dry electrodes on the headband needs training or one familiar with the EEG acquiring work. To avoid the mutual interference during the adjustment, inelastic material was used to fabricate the cap. Compared with the headband's textile material, wearing feeling of the inelastic material is worse. A preliminary test showed that adding a layer of textile or leather material inside the cap will improve the comfort feeling. The detailed material structure needs further optimization.

Since the electrode panel in the demonstration was designed and fabricated for SSVEP recording, the present cap is only fit for eight to ten electrodes on the occipital region. Other electrode panels have to be designed for EEG recording on other areas.

\section{Conclusions}

In this paper, the prototype of a kind of wireless EEG cap with an individually adjustable dry electrode on it was designed, developed, and verified. The cap was mainly composed of two hoops and an electrode panel. The hoop-structure cap and adjustable electrode handle were designed as basic units of the cap. If other panels are designed, it can be fit to record all the brain area only by changing the electrode panels, on which the number, location, and orientation angle of adjustable electrodes were installed. The quick size-adjustable hoops allows the electrode panel to be mounted well on the head in three minutes. The independently height-adjustable electrode decreased mutual interference during impedance adjusting and achieved more even impedance of the dry electrodes mounted on the cap. The proposed cap can be quickly worn and performs well in a practical SSVEP-based BCI application. The inelastic material used to fabricate the cap can be changed to a composite material to improve the wearing feeling. In adition, ultralight and soft materials may be chosen to improve comfort.

Author Contributions: Funding acquisition, W.P. and H.C.; investigation, X.W., X.H., Y.L. (Yuanyuan Liu), Y.W., W.P., C.W., Y.L. (Yamin Li), M.Y. and H.C.; methodology, X.W., L.Z., L.J., Y.L. (Yuanyuan Liu), X.X., Y.W., W.P. and X.Y.; software, X.W., L.Z., L.J., X.H., L.X. and Z.L.; supervision, W.P.; writing-original draft, X.W. and W.P.

Funding: This research was funded by National Key Technologies R\&D Program, grant number: 2017YFA0205903, 2017YFA0701100, 2016YFB0402405; National Natural Science Foundation of China, grant number: 61634006 and 61671424; The strategic priority research program of CAS pilot project, grant number: XDB32030100, XDB32040200; Key research program of frontier science, CAS, QYZDY-SSW-JSC004; CAS pilot project: XDA16020902; Beijing S\&T planning task, Z161100002616019.

Acknowledgments: The authors acknowledge the technical support from Neuracle Technology company.

Conflicts of Interest: The authors declare no conflict of interest.

\section{References}

1. Liao, L.D.; Lin, C.T.; McDowell, K.; Wickenden, A.E.; Gramann, K.; Jung, T.P.; Ko, L.W.; Chang, J.Y. Biosensor Technologies for Augmented Brain-Computer Interfaces in the Next Decades. Proc. IEEE 2012, 100, 1553-1566. [CrossRef]

2. Zander, T.O.; Lehne, M.; Ihme, K.; Jatzev, S.; Correia, J.; Kothe, C.; Picht, B.; Nijboer, F. A dry EEG-system for scientifc research and brain-computer interfaces. Front. Neurosci. 2011, 5, 53. [CrossRef] [PubMed]

3. Xing, X.; Wang, Y.; Pei, W.; Guo, X.; Liu, Z.; Wang, F.; Ming, G.; Zhao, H.; Gui, Q.; Chen, H. A High-Speed SSVEP-Based BCI Using Dry EEG Electrodes. Sci. Rep. 2018, 8, 14708. [CrossRef] [PubMed]

4. Martin, S. A high-speed brain-computer interface (BCI) using dry EEG electrodes. PLoS ONE 2017, 12, e0172400.

5. Searle, A.; Kirkup, L. A direct comparison of wet, dry and insulating bioelectric recording electrodes. Physiol. Meas. 2000, 21, 271-283. [CrossRef] [PubMed]

6. Li, G.L.; Wang, S.Z.; Duan, Y.W. Towards gel-free electrodes: A systematic study of electrode-skin impedance. Sens. Actuators B 2017, 241, 1244-1255. [CrossRef]

7. Guger, C.; Krausz, G.; Allison, B.Z.; Edlinger, G. Comparison of Dry and Gel Based Electrodes for P300 Brain-Computer Interfaces. Front. Neurosci. 2012, 6, 60. [CrossRef] 
8. Minguillon, J.; Lopez-Gordo, M.A.; Pelayo, F. Trends in EEG-BCI for daily-life: Requirements for artifact removal. Biomed. Signal Process. Control 2017, 31, 407-418. [CrossRef]

9. Mullen, T.R.; Kothe, C.A.; Chi, Y.M.; Ojeda, A.; Kerth, T.; Makeig, S.; Jung, T.P.; Cauwenberghs, G. Real-Time Neuroimaging and Cognitive Monitoring Using Wearable Dry EEG. IEEE Trans. Biomed. Eng. 2015, 62, 2553-2567. [CrossRef]

10. Chi, Y.M.; Wang, Y.T.; Wang, Y.; Maier, C.; Jung, T.P.; Cauwenberghs, G. Dry and Noncontact EEG Sensors for Mobile Brain-Computer Interfaces. IEEE Trans. Neural Syst. Rehabil. Eng. 2011, 20, 228-235. [CrossRef]

11. Löfhede, J.; Seoane, F.; Thordstein, M. Textile Electrodes for EEG Recording-A Pilot Study. Sensors 2012, 12, 16907-16919. [CrossRef] [PubMed]

12. Matthews, R.; McDonald, N.J.; Hervieux, P.; Turner, P.J.; Steindorf, M.A. A wearable physiological sensor suite for unobtrusive monitoring of physiological and cognitive state. In Proceedings of the 29th Annual International Conference of the IEEE Engineering in Medicine and Biology Society, Lyon, France, 22-26 August 2007; p. 5276.

13. Huang, Y.J.; Wu, C.Y.; Wong, A.M.K.; Lin, B.S. Novel Active Comb-shaped Dry Electrode for EEG Measurement in Hairy Site. IEEE Trans. Biomed. Eng. 2014, 62, 256-263. [CrossRef] [PubMed]

14. Liao, L.D.; Chen, B.W.; Tseng, K.C.; Ko, L.W.; Wang, I.J.; Chen, S.A.; Chang, J.Y.; Lin, C.T. Design and Implementation of Wearable and Wireless Multi-Channel Brain-Computer Interface with the Novel Dry Sensors. J. Neurosci. Neuroeng. 2013, 2, 477-484. [CrossRef]

15. Chi, Y.M.; Wang, Y.; Wang, Y.T.; Jung, T.P.; Kerth, T.; Cao, Y. A Practical Mobile Dry EEG System for Human-Computer Interfaces. In International Conference on Augmented Cognition; Schmorrow, D.D., Fidopiastis, C.M., Eds.; Springer: Berlin/Heidelberg, Germany, 2013; pp. 649-655.

16. Flumeri, G.D.; Aricò, P.; Borghini, G.; Sciaraffa, N.; Florio, A.D.; Babiloni, F. The Dry Revolution: Evaluation of Three Different EEG Dry Electrode Types in Terms of Signal Spectral Features, Mental States Classification and Usability. Sensors 2019, 19, 1365. [CrossRef] [PubMed]

17. Taji, B.; Chan, A.D.C.; Shirmohammadi, S. Effect of Pressure on Skin-Electrode Impedance in Wearable Biomedical Measurement Devices. IEEE Trans. Instrum. Meas. 2019, 67, 1900-1912. [CrossRef]

18. Wang, Y.; Wang, R.; Gao, X.; Hong, B.; Gao, S. A practical VEP-based brain-computer interface. IEEE Trans. Neural Syst. Rehabil. Eng. 2006, 14, 234-240. [CrossRef] [PubMed]

19. Muller-Putz, G.R.; Pfurtscheller, G. Control of an electrical prosthesis with an SSVEP-based BCI. IEEE Trans. Biomed. Eng. 2008, 55, 361-364. [CrossRef]

20. Garcia, G. High frequency SSVEPs for BCI applications. Available online: http://citeseerx.ist.psu.edu/ viewdoc/download?doi=10.1.1.160.879\&rep=rep1\&type=pdf (accessed on 15 May 2019).

21. Nakanishi, M.; Wang, Y.; Wang, Y.T.; Jung, T.P. A comparison study of canonical correlation analysis based methods for detecting steady-state visual evoked potentials. PLoS ONE 2015, 10, e140703. [CrossRef]

22. Neuracle. Available online: http://www.neuracle.cn/en/ (accessed on 15 May 2019).

23. Volosyak, I.; Valbuena, D.; Malechka, T.; Peuscher, J.; Gr“aser, A. Brain-Computer Interface using Water-based Electrodes. J. Neural Eng. 2010, 7, 066007. [CrossRef]

24. Fiedler, P.; Pedrosa, P.; Griebel, S.; Fonseca, C.; Vaz, F.; Supriyanto, E.; Zanow, F.; Haueisen, J. Novel Multipin Electrode Cap System for Dry Electroencephalography. Brain Topogr. 2015, 28, 5. [CrossRef]

25. DSI-24. Available online: https://wearablesensing.com/products/dsi-24/ (accessed on 15 May 2019).

26. Chen, X.G.; Wang, Y.J.; Gao, S.K.; Jung, T.P.; Gao, X.R. Filter bank canonical correlation analysis for implementing a high-speed SSVEP-based brain-computer interface. J. Neural Eng. 2015, 12, 046008. [CrossRef] [PubMed]

27. Nakanishi, M.; Wang, Y.; Chen, X.; Wang, Y.T.; Gao, X.; Jung, T.P. Enhancing Detection of SSVEPs for a High-Speed Brain Speller Using Task-Related Component Analysis. IEEE Trans. Biomed. Eng. 2018, 65, 104-112. [CrossRef] [PubMed]

28. Chien, Y.Y.; Lin, F.C.; Zao, J.K.; Chou, C.C.; Huang, Y.P.; Kuo, H.Y.; Wang, Y.; Jung, T.P.; Shieh, H.P.D. Polychromatic SSVEP stimuli with subtle flickering adapted to brain-display interactions. J. Neural Eng. 2017, 14, 016018. [CrossRef] [PubMed]

(C) 2019 by the authors. Licensee MDPI, Basel, Switzerland. This article is an open access article distributed under the terms and conditions of the Creative Commons Attribution (CC BY) license (http://creativecommons.org/licenses/by/4.0/). 